\title{
Two Expansin Genes, AtEXPA4 and AtEXPB5, Are Redundantly Required for Pollen Tube Growth and AtEXPA4 Is Involved in Primary Root Elongation in Arabidopsis thaliana
}

\author{
Weimiao Liu ${ }^{1,2}$, Liai Xu ${ }^{1,2}$ (D) Hui Lin ${ }^{3}$ and Jiashu Cao ${ }^{1,2,4, *}$ \\ 1 Laboratory of Cell and Molecular Biology, Institute of Vegetable Science, Zhejiang University, \\ Hangzhou 310058, China; 11616044@zju.edu.cn (W.L.); 11416052@zju.edu.cn (L.X.) \\ 2 Key Laboratory of Horticultural Plant Growth, Development and Quality Improvement, \\ Ministry of Agriculture, Hangzhou 310058, China \\ 3 Crop Research Institute, Fujian Academy of Agricultural Sciences, Fuzhou 350013, China; lhlz1540@163.com \\ 4 Zhejiang Provincial Key Laboratory of Horticultural Plant Integrative Biology, Hangzhou 310058, China \\ * Correspondence: jshcao@zju.edu.cn; Tel.: +86-131-8501-1958
}

check for updates

Citation: Liu, W.; Xu, L.; Lin, H.; Cao, J. Two Expansin Genes, AtEXPA4 and AtEXPB5, Are Redundantly Required for Pollen Tube Growth and AtEXPA4 Is Involved in Primary Root Elongation in Arabidopsis thaliana. Genes 2021, 12, 249. https://doi.org/ 10.3390/genes12020249

Academic Editor: Christian Chevalier Received: 8 December 2020

Accepted: 5 February 2021

Published: 10 February 2021

Publisher's Note: MDPI stays neutral with regard to jurisdictional claims in published maps and institutional affiliations.

Copyright: (c) 2021 by the authors. Licensee MDPI, Basel, Switzerland. This article is an open access article distributed under the terms and conditions of the Creative Commons Attribution (CC BY) license (https:// creativecommons.org/licenses/by/ $4.0 /)$.

\begin{abstract}
The growth of plant cells is inseparable from relaxation and expansion of cell walls. Expansins are a class of cell wall binding proteins, which play important roles in the relaxation of cell walls. Although there are many members in expansin gene family, the functions of most expansin genes in plant growth and development are still poorly understood. In this study, the functions of two expansin genes, AtEXPA4 and AtEXPB5 were characterized in Arabidopsis thaliana. AtEXPA4 and AtEXPB5 displayed consistent expression patterns in mature pollen grains and pollen tubes, but AtEXPA4 also showed a high expression level in primary roots. Two single mutants, atexpa4 and atexpb5, showed normal reproductive development, whereas atexpa4 atexpb5 double mutant was defective in pollen tube growth. Moreover, AtEXPA4 overexpression enhanced primary root elongation, on the contrary, knocking out AtEXPA4 made the growth of primary root slower. Our results indicated that AtEXPA4 and AtEXPB5 were redundantly involved in pollen tube growth and AtEXPA4 was required for primary root elongation.
\end{abstract}

Keywords: Arabidopsis thaliana; expansin genes; AtEXPA4; AtEXPB5; pollen tube growth; root elongation

\section{Introduction}

Plant growth and development are inseparable from cell proliferation and expansion. Different from animal cells, plant cells are surrounded by cell walls, which are highly dynamic and complex networks [1]. Cells are always in a dynamic balance between the expansion of protoplasts and the restraint of cell walls, undergoing irreversible growth [2]. Although cell walls expand slowly while plants grow, some organs and tissues require rapid expansion of cell walls during specific periods, such as fast-growing roots and pollen tubes.

Numerous cell wall synthesis and remodeling genes have been reported to be involved in cell wall expansion [3-9], including expansin genes. Expansins can weaken non-covalent bonds between cell wall polysaccharides, thereby promoting slippage and relaxation of cellulose microfibers, resulting in cell wall extension and cell expansion [10,11]. Expansins usually have two typical domains, DPBB_1 and Pollen_allerg_1, and most of expansins have signal peptides [12]. DPBB_1 domain is often related to family-45 glycosyl hydrolase (GH45), and contains a conserved region that has the double-psi beta-barrel (DPBB) fold $[13,14]$. Although DPBB_1 is similar to GH45, expansins lack the $\beta$-1,4-glucanase activity of GH45 enzymes, which in turn lacks the wall expanding activity of expansins [15]. Pollen_allerg_ 1 is often a pollen allergen, which is found at the C-terminus of expansins [16]. The expansin gene family with many members can be divided into two major subfamilies, namely EXPA and EXPB subfamilies [15]. The various temporal and spatial expression 
patterns of expansin genes imply that they perform corresponding functions in different developmental stages [12,17-19]. Many studies have shown that EXPA subfamily plays complex and diverse functions in plant growth and development. For example, Arabidopsis thaliana EXPA2 (AtEXPA2) participates in seed germination under the regulation of upstream transcription factors [20-22]. AtEXPA5 participates in plant growth and development regulated by ethylene and brassinosteroids $[23,24]$. AtEXPA5 and Oryza sativa EXPA8 (OsEXPA8) $[25,26]$ are both involved in the development of primary roots. The inhibition of their expressions reduces the length of primary root. Moreover, AtEXPA14 and AtEXPA17 participate in the growth of lateral roots and root hairs by the regulation of auxin-related transcription factor LBD18 [27,28].

However, researches on the roles of expansins in plant reproductive development are relatively limited. It was only confirmed that Zea mays EXPB1 (ZmEXPB1) showed a great influence on the growth of pollen tubes in vivo and positively affected the entry of pollens into ovules [29-31].

A previous study showed that two expansin genes, AtEXPA4 and AtEXPB5, were strongly expressed in dry pollen grains, imbibed pollen grains, and pollen tubes [32-36]. Here, we further confirmed the expression patterns of AtEXPA4 and AtEXPB5. By exploring phenotypes of mutants and overexpression lines, we found that AtEXPA4 and AtEXPB5 are redundantly involved in pollen tube elongation, and AtEXPA4 plays a positive role in primary root growth.

\section{Materials and Methods}

\subsection{Plant Materials and Growth Conditions}

All transgenic plants used in this study were of Arabidopsis thaliana (A. thaliana) Columbia ecotype (Col-0) background and were obtained by Agrobacterium tumefaciensmediated floral dip method [37]. The homozygous mutants atexpa4 and atexpb5 were obtained by CRISPR/Cas9 system [38]. Two off-target sites of AtEXPA4 and AtEXPB5 were predicted by the CRISPR-P 2.0 website (http:/ / crispr.hzau.edu.cn/CRISPR2/ (accessed on 1 February 2020)), respectively. To obtain heterozygous double mutants of AtEXPA4 and AtEXPB5, atexpb5 and atexpa4 homozygous plants were used as female and male parents in a cross. The homozygous double mutants, atexpa4expb5, were generated by self-crossing with heterozygous $\mathrm{F}_{1}$ plants. The genotypes of $362 \mathrm{~F}_{2}$ plants were confirmed by PCR and sequencing, and genotype statistics and analysis were performed.

A 1522-bp promoter sequence and the coding sequence of AtEXPA4 (splicing variant: At2g39700.1) were amplified from Col-0 genomic DNA and inflorescence cDNA. A 1571-bp promoter sequence and the coding sequence of AtEXPB5 (splicing variant: At3g60570.1) were also amplified from Col-0 genomic DNA and inflorescence cDNA. Then they were subcloned into pBI101 vectors to create the fusion overexpression constructs proAtEXPA4::EXPA4, proAtEXPB5::EXPB5 and the promoter analysis construct proAtEXPA4::GUS, proAtEXPB5::GUS. The complemented lines were obtained by transforming proAtEXPA4::EXPA4 and proAtEXPB5::EXPB5 into the double mutant atexpa4expb5, and named atexpa4expb5 $5^{A 4 O E}$ and atexpa4expb5 $5^{B O E}$, respectively. The homozygous overexpression lines AtEXPA4 ${ }^{\mathrm{OE}}, A t E X P B 5^{\mathrm{OE}}$ and promoter analysis lines of AtEXPA4, AtEXPB5 were identified from screening $\mathrm{T}_{3}$ plants on plates containing kanamycin $\left(50 \mathrm{mg} \cdot \mathrm{L}^{-1}\right)$. Two complementary lines were identified from screening $\mathrm{T}_{1}$ plants on plates containing kanamycin $\left(50 \mathrm{mg} \cdot \mathrm{L}^{-1}\right)$. All seedlings were transplanted into soil, and grown in a $22{ }^{\circ} \mathrm{C}$ climate chamber under long-day conditions (16 h light/8 h dark). All the primers were listed in Table S1.

For root phenotype analysis, the seeds of wild-type, atexpa4, atexpb5, AtEXPA4 ${ }^{O E}$, and $A t E X P B 5^{O E}$ were surface-sterilized with $75 \%$ ethanol for $5 \mathrm{~min}$ and washed 5 times with sterile water. Subsequently, these seeds were geminated on 1/2 Murashige and Skoog solid media and placed horizontally at $4{ }^{\circ} \mathrm{C}$ for 3 days. Next, plates were transferred to an artificial climate chamber and placed vertically on the platform, and then germinated at $22 \pm 1{ }^{\circ} \mathrm{C}$ under $12 \mathrm{~h}$ light/12 $\mathrm{h}$ dark cycle. 


\section{2. $m R N A$ Expression Analysis}

Trizol reagent (Invitrogen, Carlsbad, CA, USA) were used to extract total RNA from plant tissues and first-strand cDNA was synthesized by PrimerScript RT reagent Kit (TaKaRa, Kyoto, Japan) from $1 \mu \mathrm{g}$ total RNA. The relative expression levels of corresponding genes in different tissues and organs were detected by quantitative real-time PCR (qRT-PCR), which were performed by using TaKaRa TB Green ${ }^{\mathrm{TM}}$ Premix Ex Taq $^{\mathrm{TM}}$ II (Tli RNaseH Plus) on a Real-Time PCR machine (CFX96 Real-Time System, Bio-Rad, Carlsbad, CA, USA). Primers for AtEXPA4 and AtEXPB5 were listed in Supplemental Table S1. BETA-TUBULIN4 (At5g44340) was used as the reference gene for AtEXPA4 and AtEXPB5 expression in different tissues and different transgenic lines. All experiments were performed three biological replicates, and each biological replicate was performed three technical replicates. Moreover, transcript levels of target genes were calculated relative to BETA-TUBULIN4 using the $2^{-\Delta \Delta \mathrm{Ct}}$ method.

\subsection{Histochemical GUS Staining Assay to Analyze Promoter Activity}

The promoter analysis constructs, proAtEXPA4::GUS and proAtEXPB5::GUS, were transferred into wild-type plants by Agrobacterium tumefaciens-induced floral dip method. More than six independent $T_{1}$ lines were screened continuously until $\mathrm{T}_{3}$ homozygous plants were obtained. Seedlings and inflorescences from homozygous plants were used to detect GUS activity. The inflorescences from 35-day-old plants and seedlings at different stages were stained with GUS working solution (GUS working solution: X-Gluc solution and Basic solution $(1: 9, v / v)$. X-Gluc solution: dissolving X-Gluc with $\mathrm{N}-\mathrm{N}$-dimethylamide (DMF) to make a $20 \mathrm{mM}$ solution. Basic solution: $50 \mathrm{mM} \mathrm{NaH}{ }_{2} \mathrm{PO}_{4}, 50 \mathrm{mM} \mathrm{Na}_{2} \mathrm{HPO}_{4}, 10 \mathrm{mM}$ $\mathrm{Na}_{2}$ EDTA, $0.1 \%(v / v)$ Triton X-100, $\left.0.5 \mathrm{mM} \mathrm{K}_{3}\left[\mathrm{Fe}(\mathrm{CN})_{6}\right]\right)$, and $0.5 \mathrm{mM} \mathrm{K}_{4}\left[\mathrm{Fe}(\mathrm{CN})_{6}\right]$.) and then incubating in dark at $37{ }^{\circ} \mathrm{C}$ overnight. Tissues were decolorized in $75 \%$ and $90 \%$ ethanol in order, and images were taken by a differential interference microscopy (Nikon, Tokyo, Japan). Each tissue staining contained at least three biological replicates. Moreover, the stage of flower development was confirmed by previous studies [39,40].

\subsection{Subcellular Localizations of AtEXPA4 and AtEXPB5}

To observe subcellular distributions of AtEXPA4 and AtEXPB5, we amplified the AtEXPA4 and AtEXPB5 coding sequences with gene-specific primers (Table S1), and then subcloned these into a vector containing the enhanced green fluorescent genes $(e G F P)$, to form pro35S::AtEXPA4::eGFP and pro35S::AtEXPB5::eGFP constructs. The fusion vectors were transiently transformed into onion epidermal cells using a helium-driven accelerator (PDS/1000, Bio-Rad). The parameters were as follows: $1 \mu \mathrm{m}$ gold particles, $1100 \mathrm{psi}$ bombardment pressure, and a distance of $9 \mathrm{~cm}$ from microcarrier to samples. After $24 \mathrm{~h}$ of cultivation, the onion epidermal cells with $e G F P$ expression were observed and pictures were taken by a fluorescence microscope (ECLIPSE 90i, Nikon). To visualize eGFP distribution, onion epidermal cells were plasmolyzed in $0.1 \mathrm{~g} \cdot \mathrm{mL}^{-1}$ sucrose solution for $5 \mathrm{~min}$. For subcellular localization experiments using tobacco (Nicotiana benthamiana), the fusion vectors were transiently transformed into leaf epidermal cells by the infiltrated method. After $48 \mathrm{~h}$ of introduction, the subcellular localization of eGFP-fusion protein was analyzed by a confocal laser scanning microscope (A1, Nikon) with the NIS-elements AR software version 4.60(Nikon).

The subcellular localization experiments of AtEXPA4 and AtEXPB5 have been carried out using at least three biological replicates, and no less than 5 cells with eGFP signal were observed in each replicate.

\subsection{Analysis of Primary Root and Meristem Length}

To observe primary root growth, the 3, 5, 7-day-old seedlings were taken photos by a stereo microscope (Leica, Germany). Subsequently, the primary root length was measured ranging from the base of hypocotyl to the root tip by ImageJ 1.52a software (https:/ /imagej.nih.gov/ij/ (accessed on 29 March 2020)). 
The length of root meristem was defined as the distance from quiescent center $(\mathrm{QC})$ to transition zone (TZ, that is the position of the first elongating cortical cell) [4]. To measure the length of root meristems, roots were fixed in saturated chloral hydrate and images were taken by a differential interference microscopy (Nikon, Japan). All experiments were performed in three biological replicates with at least 12 seedlings measured in each replicate.

\subsection{Phenotype Analysis of Pollen and Pollen Tube}

Alexander staining was used to detect pollen vitality [41]. Mature pollen grains that develop normally will be dyed red or purple, and abnormal or immature pollen grains will be dyed blue or green. 4',6-Diamidino-2-phenylindole (DAPI) staining was used to detect whether pollen nuclei were normal [42]. Under ultraviolet light, normal pollen nuclei (two sperm nuclei and one nutrient nucleus) will produce intense fluorescence. Aniline blue staining could detect callose development and degradation [43]. In tetrad stage, microspores are wrapped by callose wall, which can combine with the aniline blue dye to produce strong fluorescence under ultraviolet illumination. Under normal circumstances, the callose wall gradually degrades with the development of pollen. In mature pollen stage, the callose wall has been completely degraded and only the weak fluorescence of pollen grains can be observed but no strong fluorescence will appear under ultraviolet light. All micrographs were taken by an inverted fluorescence microscope (Nikon, Japan). Scanning electron microscope (SEM) observation and pollen germination in vivo were conducted as described in Lin et al. [44]. The mature pollens and tetrads were peeled off from flower buds in stage 13 and stage $6-8$ of 35-d-old inflorescences, respectively. Each kind of staining was repeated three times, using at least 5 plants each time, and observing no less than 30 fields of view. The length of pistil and pollen tube was measured by ImageJ, and the ratio of pollen tube to pistil length was calculated as an indicator of the germination degree of pollen tube. The experiments were carried out using three biological replications with at least 7 pistils measured in each replicate.

\section{Results}

\subsection{Analysis of AtEXPA4 and AtEXPB5 Expression Patterns}

Through the analysis of domains on the Pfam website (http:/ / www.sanger.ac.uk/ Software/Pfam/ (accessed on 21 March 2018)), we confirmed that both AtEXPA4 and AtEXPB5 contain DPBB_1 and Pollen_allerg_1, which have typical domains of the expansin gene family (Figure S1) [12].qRT-PCR and GUS reporting system were used to explore spatial and temporal expression of them. The results showed that AtEXPA4 seemed to expressed ubiquitously (Figure 1A), but its expression level was higher in inflorescences and roots (Figure 1A,B). In inflorescences, AtEXPA4 was expressed from the stage 11 of floral buds to the mature pollen stage. Subsequently, strong GUS signal appeared in pollen tubes, which indicated that AtEXPA4 was also highly expressed during pollen tube growth (Figure 1C). Further research found that AtEXPA4 was expressed in the root tips, especially in root meristems of 3-d-old seedlings. As time passed, AtEXPA4 was expressed in the entire root by the 7th day after seed germination (Figure 1D). As for AtEXPB5, the qRT-PCR result showed that $A t E X P B 5$ was predominantly expressed in inflorescences and siliques, but weakly expressed in other tissues (Figure 1E,F). GUS staining also confirmed that AtEXPB5 was highly expressed in inflorescences. Moreover, GUS signal in pollen tube was very significant, suggesting that $A t E X P B 5$ was strongly expressed during pollen tube growth (Figure 1G). 


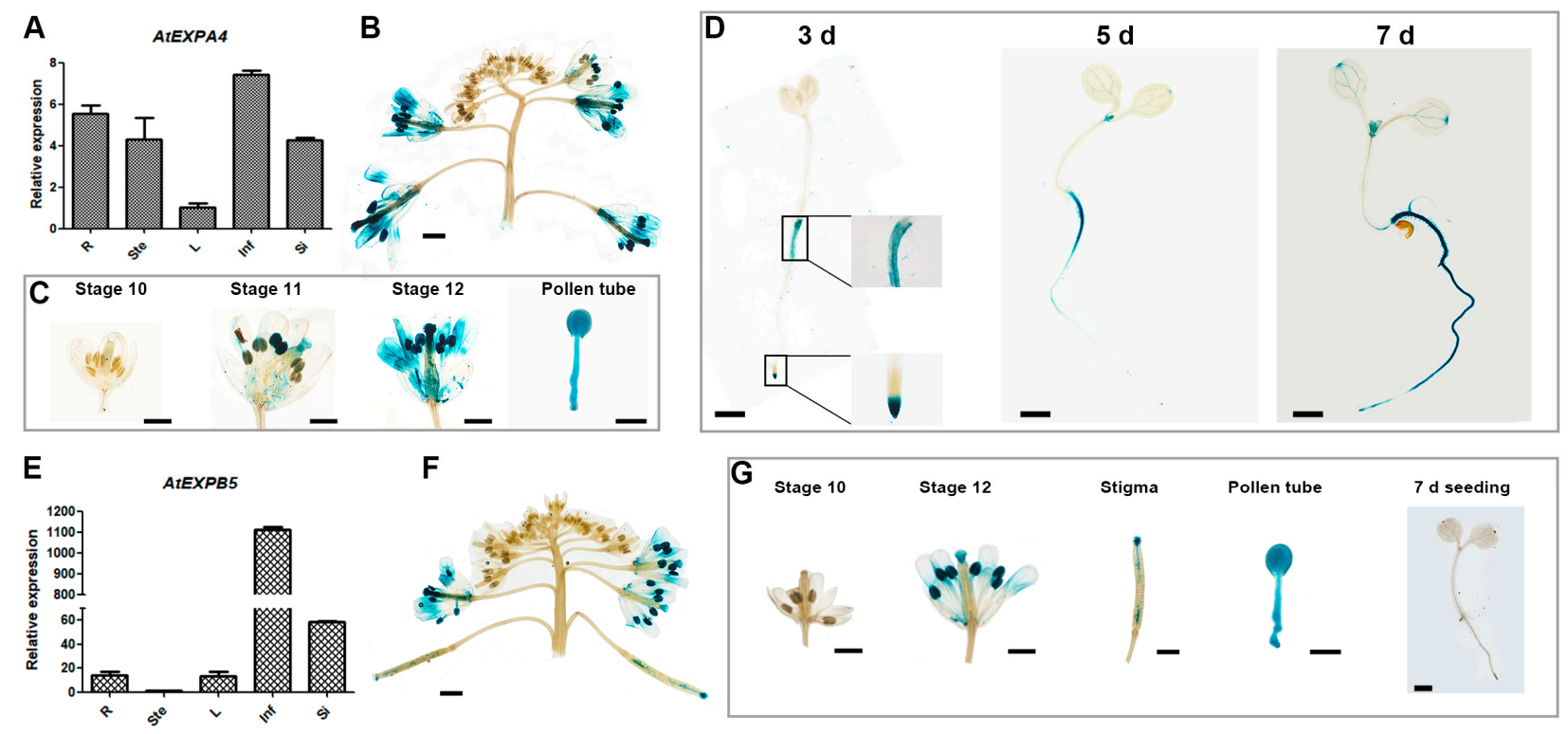

Figure 1. Expression pattern analysis of AtEXPA4 and AtEXPB5. (A,E) qRT-PCR analysis of AtEXPA4 (A) and AtEXPB5 (E) transcripts in different tissues of Arabidopsis thaliana: 35-d-old roots (R), 35-d-old stems (Ste), young rosette leaves (L), 35-d-old inflorescences (Inf) and siliques (Si). BETA-TUBULIN4 was used as the reference gene. AtEXPA4 expression in leaf and AtEXPB5 expression in stem were normalized to 1 . The values are the mean $\pm \mathrm{SD}$ (standard deviation), three biological replicates with three technical replicates in each biological replicate. (B-D) Analysis of proAtEXPA4::GUS activity. (B) GUS activity in inflorescences. (C) GUS activity in different developmental stages of buds and pollen tubes. (D) GUS activity in seedlings at different time points after germination, days (d). (F,G) Analysis of proAtEXPB5::GUS activity. (F) GUS activity in inflorescences. (G) GUS activity in different developmental stages of buds, stigmas, pollen tubes, and 7-d-old seedlings. Scale bars of pollen tubes, $25 \mu \mathrm{m}$. Scale bars of others, $1 \mathrm{~mm}$.

\subsection{AtEXPA4 and AtEXPB5 Exhibit Cell Wall Localizations}

To test whether AtEXPA4 and AtEXPB5 were cell wall binding proteins, eGFP genes were fused to AtEXPA4 and AtEXPB5 coding sequences to form AtEXPA4::eGFP and AtEXPB5::eGFP constructs under the control of constitutive CaMV $35 S$ promoter. Subsequently, these constructs were transiently transformed into onion and tobacco epidermal cells. The eGFP fluorescence in tobacco leaf epidermal cells appeared in nuclei and plasma membrane, which indicated that AtEXPA4 and AtEXPB5 localized in nuclei and plasma membrane (Figure S2). Furthermore, the eGFP fluorescent signal associated with AtEXPA4 and AtEXPB5 localized homogeneously in unplasmolyed transformed onion cells, including cytoplasm and nuclei (Figure 2E,F,I,J). In plasmolyed cells, the eGFP fluorescence not only appeared in cytoplasm, nuclei, and cell membrane, but also distributed on the cell wall (Figure 2G,H,K,L). On the contrary, there was no eGFP signal on the cell wall in the control cells after plasmolysis (Figure 2C,D). These results confirmed that AtEXPA4 and AtEXPB5 exhibited cell wall localizations.

\subsection{Identification of atexpa4, atexpb5, atexpa4expb5 Mutants, and AtEXPA4 ${ }^{O E}$, AtEXPB5 ${ }^{O E}$ Lines}

In order to characterize the functions of AtEXPA4 and AtEXPB5 in plant growth and development, gene knockout and overexpression methods were used. The promoter of Cas 9 was replaced with YAOZHE promoter (proAtYAO) for improving the editing efficiency of CRISPR/Cas9 system (Figure S3A) [45]. Two sgRNAs targeting AtEXPA4 or AtEXPB5 were designed respectively to obtain knockout lines. For AtEXPB5, both sgRNAs worked, while only sgRNA11 of AtEXPA4 worked (Figures 3A and S4). Subsequently, homozygous lines atexpa4-6/47/149 and atexpb5-4/8/9 were used for phenotypic observation (Figure 3B,C). We also checked whether these lines were off-target by PCR and sequencing methods. The results showed that none of the 33 atexpa 4 and 47 atexpb5 plants at off-target sites 
were edited (Tables S2 and S3). Moreover, homozygous double mutant atexpa4expb5 was screened by PCR and sequencing (Figure S5). In addition, AtEXPA4 and AtEXPB5 were

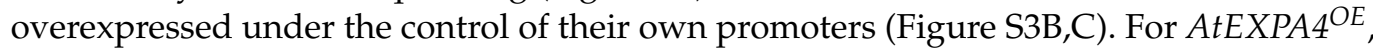
seven overexpression lines were generated and they all showed 5.8-37.1-fold increase in roots and inflorescences compared with wild-type.
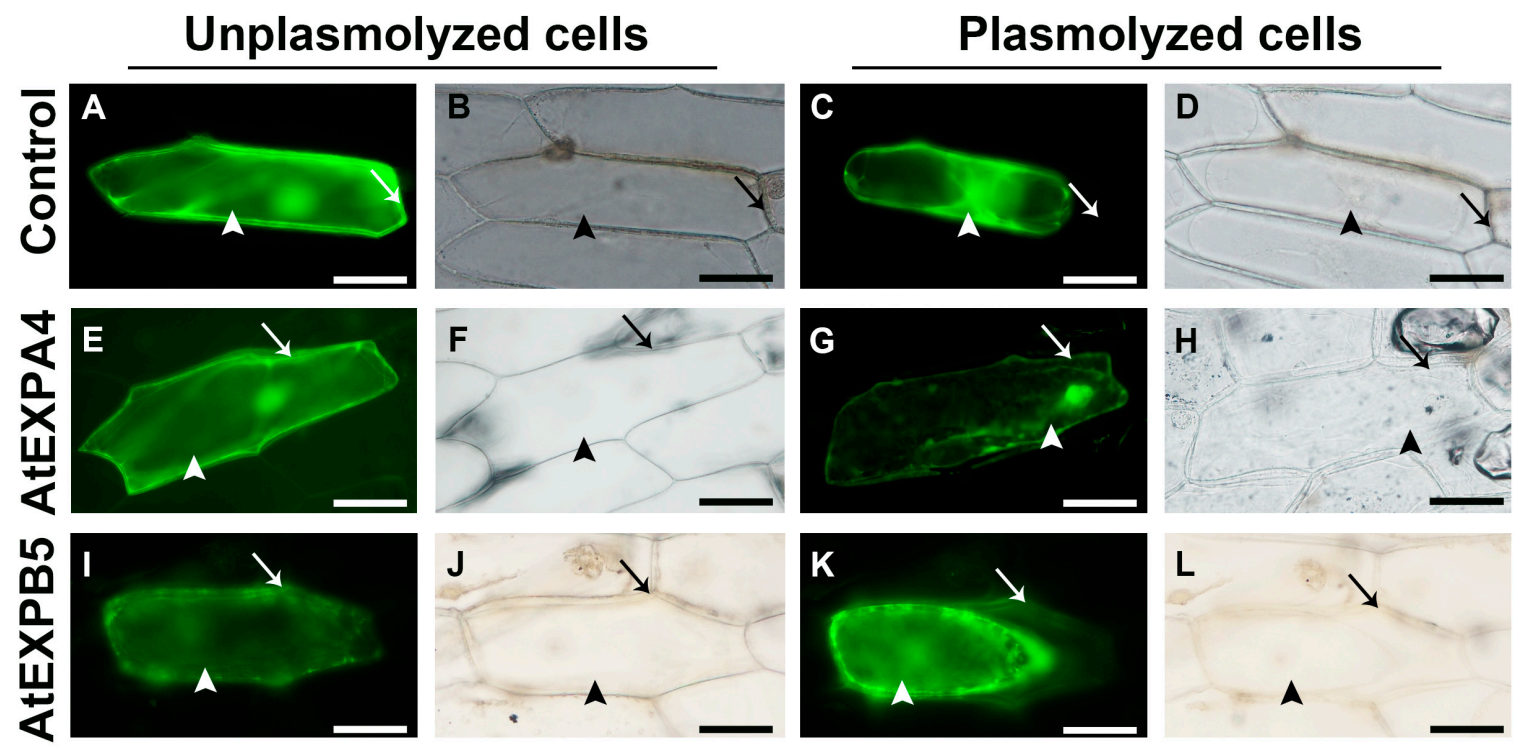

Figure 2. Subcellular localizations of AtEXPA4::eGFP and AtEXPB5::eGFP fusion proteins in onion epidermal cells. (A-D), Control cells with eGFP signals. (E-H), Onion epidermal cells with AtEXPA4::eGFP fusion signals. (I-L), Onion epidermal cells with AtEXPB5::eGFP fusion signals. (A,C,E,G,I,K), Fluorescence images. (B,D,F,H,J,L), Brightfield images. $(\mathbf{A}, \mathbf{B}, \mathbf{E}, \mathbf{F}, \mathbf{I}, \mathbf{J})$, Unplasmolysed cells. (C,D,G,H,K,L), Plasmolysed cells. Arrows and arrow heads indicate cell walls and cytoplasm, respectively. Scale bars, $100 \mu \mathrm{m}$.
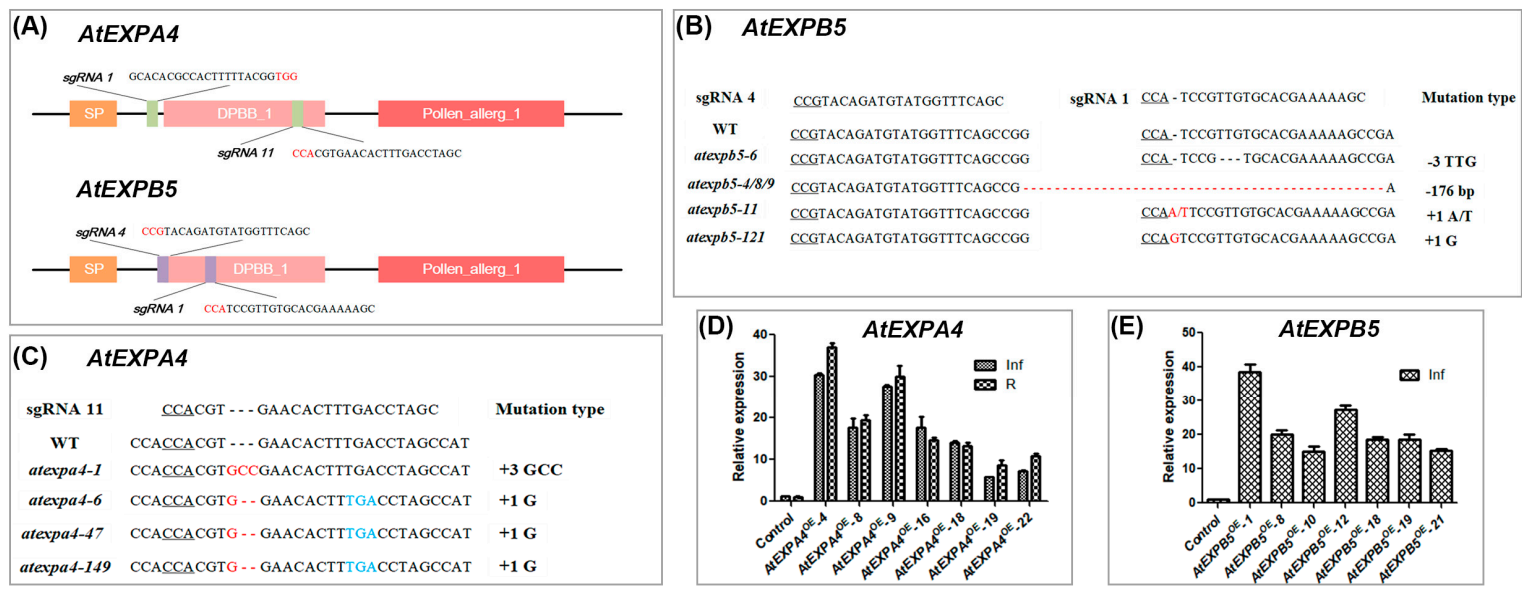

Figure 3. Confirmation of AtEXPA4 and AtEXPB5 transgenic plants. (A), Genomic location of sgRNAs targeting to AtEXPA4 and AtEXPB5. PAM sites are highlighted in red. (B,C), the gene editing situation of AtEXPA4 and AtEXPB5 by CRISPR/Cas9 system in $\mathrm{T}_{3}$ plants. Underlined letters, red letters, and blue letters represent PAM sites, gene editing sites, and stop codons, respectively. (D), qRT-PCR analysis of AtEXPA4 expression in AtEXPA4 ${ }^{\mathrm{OE}}$ lines. 35-d-old roots (R) and inflorescences (Inf). BETA-TUBULIN4 was used as the reference gene. AtEXPA4 expression in control lines were normalized to 1. (E), qRT-PCR analysis of AtEXPB5 expression in AtEXPB5 ${ }^{O E}$ lines. 35-d-old inflorescences (Inf). BETA-TUBULIN4 was used as the reference gene. AtEXPB5 expression in control lines were normalized to 1 . The values are the mean $\pm \mathrm{SD}$, three biological replicates with three technical replicates in each biological replicate. 
As for $A t E X P B 5^{O E}$, a total of 7 overexpression lines were obtained, which showed an increase of 15.0-38.5-fold compared with the wild type. The growth of homozygous lines with the highest overexpression, $A t E X P A 4^{O E_{-}} 4$ and $A t E X P B 5^{O E}-1$ were subsequently monitored (Figure 3D,E).

\subsection{Varied Expressions of AtEXPA4 and AtEXPB5 Show No Effect on Plant Morphology and Pollen Vitality}

The results of morphological observation showed that whether AtEXPA4 and AtEXPB5 were overexpressed or knocked out, plant overall morphology was not affected. Moreover, sepals, petals, stamens and pistils were not significantly abnormal compared with wildtype. Also, double mutant, atexpa4expb5, was not different from control lines (Figure S6).

Furthermore, pollen development of transgenic plants was observed by cytological staining. Alexander staining revealed that pollen grains produced by these transgenic plants all showed high vitality. By aniline blue staining, they all showed normal thickening of callose during the tetrad stage, and no abnormality in callose degradation during the subsequent pollen maturity stage. DAPI staining was used to observe pollen nuclei, and the result displayed that all transgenic pollen nuclei were development normally. SEM observation revealed that pollen morphology and surface decoration of all transgenic lines were no different from those of wild-type. (Figure S7). These results indicated that the single and double mutations and overexpression of AtEXPA4 and/or AtEXPB5 had no effect on pollen development.

\subsection{AtEXPA4 and AtEXPB5 Are Redundantly Required for Pollen Tube Growth}

To investigate the impacts of AtEXPA4 and AtEXPB5 on pollen germination, in vivo germination assay was performed. After $3 \mathrm{~h}$ of pollen germination, wild-type pollen tube length accounted for $34.59 \%$ of total pistil length, while the pollen tube length of atexpa4expb5 only accounted for $17.04 \%$ of pistil length. The pollen tube length of atexpa4expb5 was $50.74 \%$ less than that of wild-type (Figure $4 \mathrm{~A}, \mathrm{~B}$ ). With pollen tube development, the differences in pollen tube length between double mutants and wild-type became smaller than that in the $3 \mathrm{~h}$ after pollen germination, but they were still at significant levels. (Figure 4B). By $24 \mathrm{~h}$ after pollination, wild-type pollen tubes have been extended to the bottom of pistils, while pollen tube length of double mutants have only grown to $76.45 \%$ of pistil (Figure 4A,B). These results indicated that the pollen tube elongation of atexpa4expb5 was significantly slower than that of wild-type after self-pollination. However, no difference was observed in atexpa 4 and atexpb5 when compared with wild-type at various time points after pollination (Figure 4). However, the overexpression of AtEXPA4 and AtEXPB5 had no significant effect on pollen germination and elongation (Figure S8).

In addition, we investigated whether AtEXPA4 or AtEXPB5 could complement the double mutant phenotype by observing the pollen germination in vivo of complementary lines. The results of qRT-PCR showed that expression levels of AtEXPA4 and AtEXPB5 in corresponding complementary lines were increased (Figure $5 \mathrm{~A}, \mathrm{~B}$ ). We selected the highest expression lines atexpa4expb5 ${ }^{A 4 O E_{-}}-18 / 20 / 22$ and atexpa4expb5 ${ }^{B 5 O E_{-4}} / 15 / 17$ for pollen germination in vivo. The results indicated that whether transformed the double mutants with AtEXPA4 or AtEXPB5 could complement the slow pollen tube growth (Figure 5C,D).

Nevertheless, the silique and seed development of either two single mutants or double mutant were not different from that of wild-type (Figure S6), which implied that knocking out AtEXPA4 and AtEXPB5 simultaneously did not affect the transmission of sperm cells and double fertilization process. We also calculated and analyzed the segregation ratio of $\mathrm{F}_{2}$ plants produced by self-crossing of $\mathrm{F}_{1}$ plants $[\operatorname{expa4}(+/-) \operatorname{expb5}(+/-)]$, and the results showed that the slower growth did not cause expansin deficient pollen to be less competitive than wild-type pollen (Table 1). In summary, AtEXPA4 and AtEXPB5 were redundantly involved in pollen tube germination and elongation, but showed no effect on silique and seed development. 


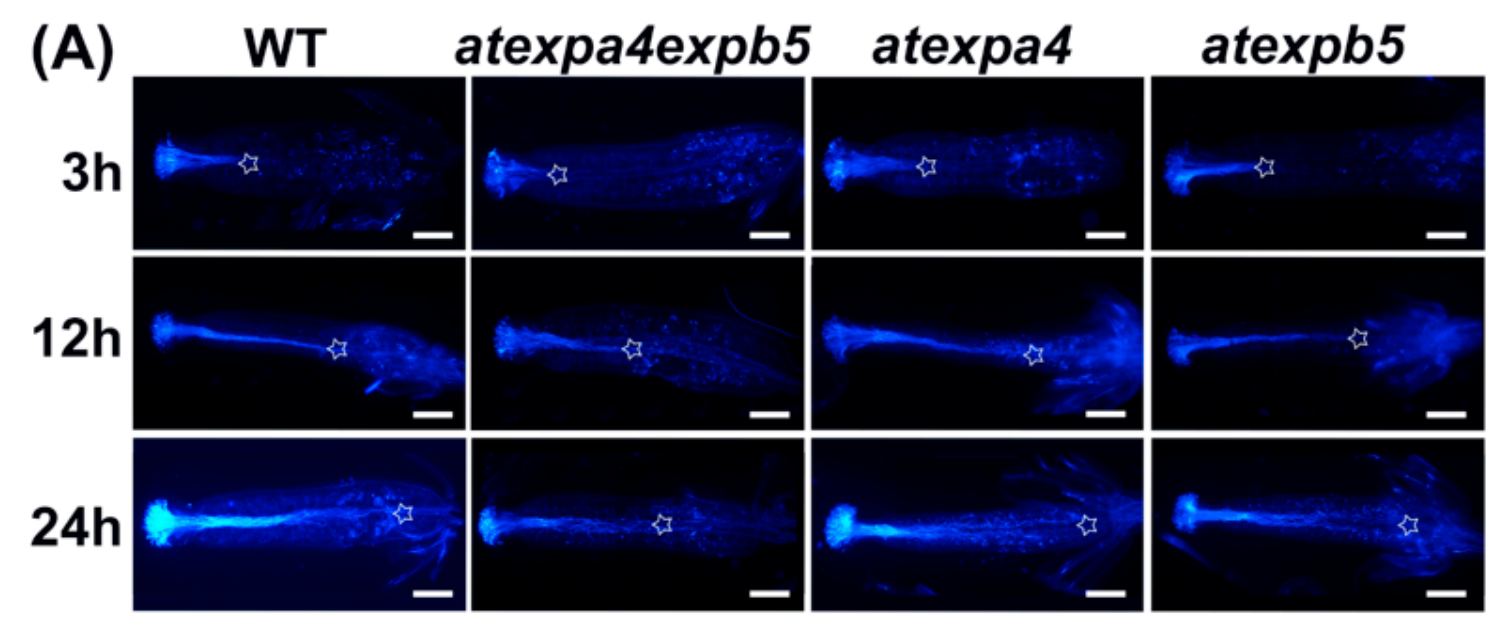

(B)

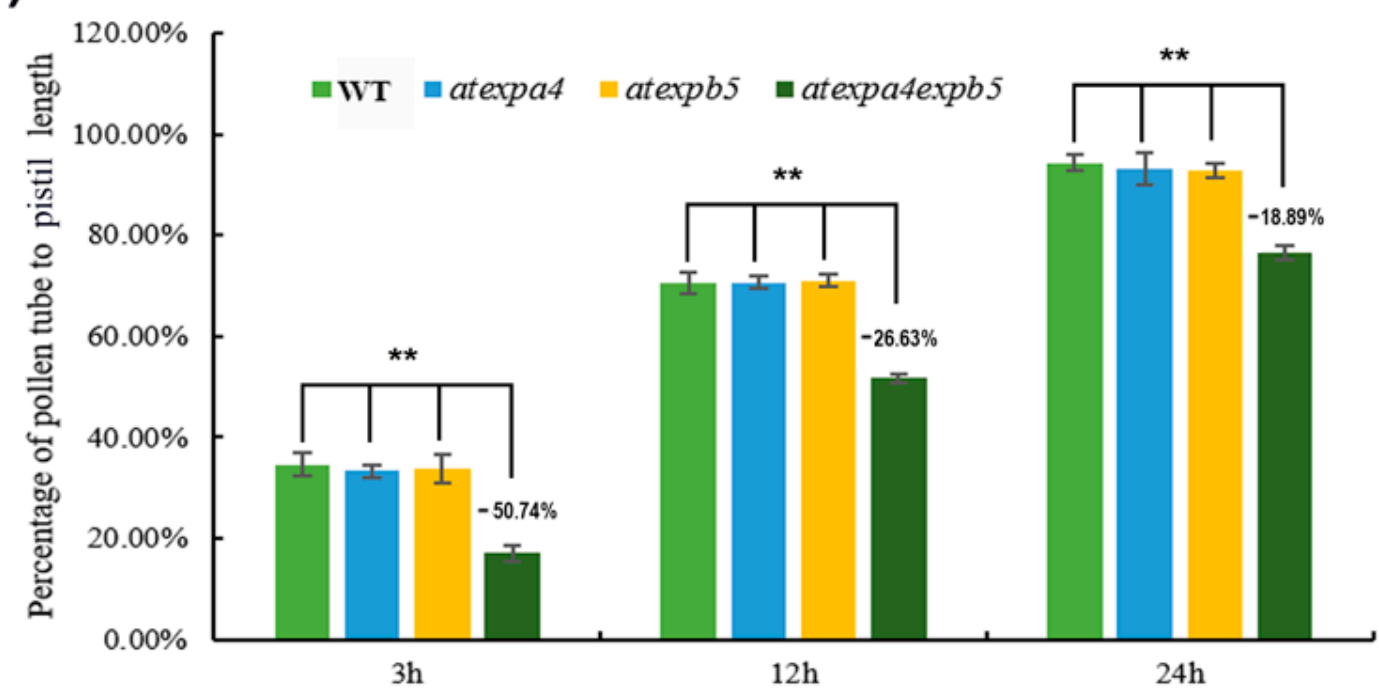

Figure 4. AtEXPA4 and AtEXPB5 are involved in pollen tube growth. (A), Aniline blue staining of pollen tubes at $3 \mathrm{~h}, 12 \mathrm{~h}$, and $24 \mathrm{~h}$ after pollination in single and double mutants, hours (h). Asterisks show positions where pollen tubes arrive, scale bars, $400 \mu \mathrm{m}$. (B), the percentage of pollen tube to pistil length in (A). Values are means, error bars are SD, $n=12$ pistils per replicate, three biological replicates, $t$-tests as ${ }^{* *} p<0.01$.

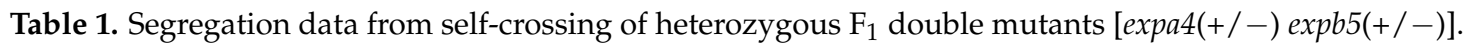

\begin{tabular}{|c|c|c|c|c|c|}
\hline \multirow{2}{*}{ Genotype of $F_{2}$ Plants } & \multirow{2}{*}{$\begin{array}{c}\text { The Observed } \\
\text { Number of } F_{2} \text { Plants }\end{array}$} & \multirow{2}{*}{$\begin{array}{c}\text { The Expected } \\
\text { Number of } F_{2} \text { Plants }\end{array}$} & \multirow{2}{*}{ Degrees of Freedom } & \multicolumn{2}{|c|}{$\chi^{2}$ Test for 9:3:3:1 ${ }^{a}$} \\
\hline & & & & $x^{2}$ & Expected $\chi^{2}(p<0.05)$ \\
\hline $\operatorname{expa4}(+/) \operatorname{expb5(+/)}$ & 196 & 203.625 & & & \\
\hline $\operatorname{expa} 4(-/-) \operatorname{expb5}(+/)$ & 76 & 67.875 & & & \\
\hline $\operatorname{expa4}(+/) \operatorname{expb5}(-/-)$ & 75 & 67.875 & 3 & $4.576^{\mathrm{b}}$ & 7.815 \\
\hline $\operatorname{expa4}(-/-) \operatorname{expb5}(-/-)$ & 15 & 22.625 & & & \\
\hline
\end{tabular}




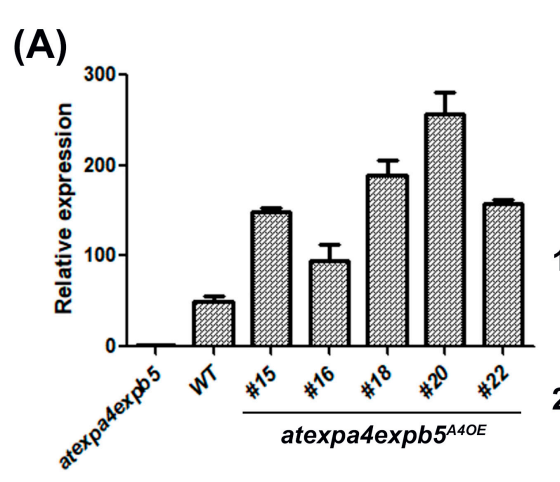

(C)
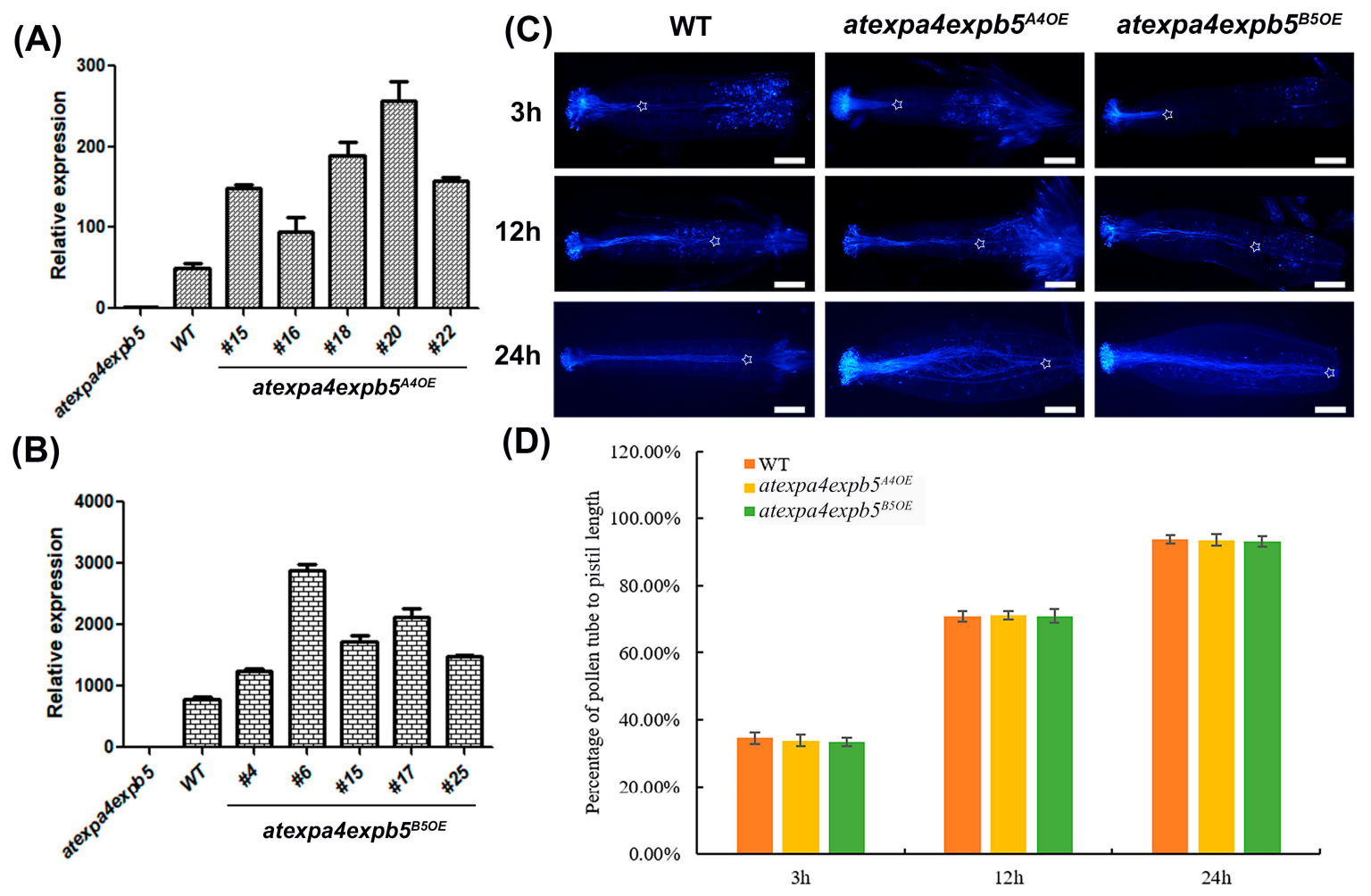

Figure 5. AtEXPA4 or AtEXPB5 can complement the slow pollen tube growth. (A,B), qRT-PCR analysis of $A t E X P A 4$ (A) or $A t E X P B 5$ (B) expression in 35-d-old inflorescences from atexpa4expb5, atexpa4expb5 $5^{A 4 O E}$, and atexpa4expb5 $5^{B 5 O E}$. BETATUBULIN4 was used as the reference gene, and expression in atexpa4expb5 was normalized to 1 . The values are the mean $\pm \mathrm{SD}$, three biological replicates with three technical replicates in each biological replicate. (C) Aniline blue staining of pollen tubes at $3 \mathrm{~h}, 12 \mathrm{~h}$, and $24 \mathrm{~h}$ after pollination, hours (h). Asterisks show positions where pollen tubes arrive, scale bars, $300 \mu \mathrm{m}$. (D) the percentage of pollen tube to pistil length in (C). Values are means, error bars are SD, $n=7$ pistils per replicate, three biological replicates.

\subsection{AtEXPA4 Participates in Primary Root Elongation}

The increasing expression of AtEXPA4 in primary roots during seedling development suggested that it might be involved in root growth. Therefore, we measured the primary root length of seedlings. Interestingly, when compared with the wild-type at $3 \mathrm{~d}, 5 \mathrm{~d}$, and $7 \mathrm{~d}$ after seed germination, the length of atexpa 4 primary root decreased by $30.76 \%, 21.92 \%$, and $37.24 \%$, respectively. On the contrary, AtEXPA4 ${ }^{\mathrm{OE}}$ seedlings exhibited increased primary root length by $16.52 \%$ and $9.98 \%$ at $3 \mathrm{~d}$ and $5 \mathrm{~d}$ after seed germination. Although the primary root length of AtEXPA4 ${ }^{O E}$ only increased by $5.67 \%$ compared with the wild-type at the 7 th day after germination, it still reached a significant level. (Figure 6A,B). Moreover, the mutation and overexpression of AtEXPB5 did not affect the growth and development of roots (Figure S9A,B). Notably, the change pattern of root meristem size was similar to that of root length. The meristem length of atexpa 4 was reduced by $25.41 \%, 18.26 \%$, and $33.00 \%$ compared with the wild-type after 3, 5, and 7 days of seed germination (Figure 7A,B). By contrast, the overexpression of AtEXPA4 led root meristems to increase by $12.15 \%, 11.27 \%$, and $7.51 \%$ at $3 \mathrm{~d}, 5 \mathrm{~d}$, and $7 \mathrm{~d}$ after seed germination (Figure $7 \mathrm{~A}, \mathrm{C}$ ).

In addition, the primary root growth rates of wild-type, atexpa4, and $A t E X P A 4^{O E}$ were similar from the 3rd day to the 5th day after germination (Figure 6C). However, some changes occurred from the 5th day to the 7th day after germination. On the one hand, primary root growth rates of wild-type and AtEXPA4 ${ }^{O E}$ were still consistent but higher than before. On the other hand, the primary root growth rate of atexpa4 was slower than that of wild-type and AtEXPA4 ${ }^{O E}$ (Figure 6C). The change pattern of meristem growth rate was consistent with that of root elongation rate. That is, the meristem growth rate of each transgenic line was closely resembled in the first 5 days after seed germination, 
while the growth rate of atexpa 4 was significantly reduced from the 5th day to the 7th day (Figure 7A,C). These findings suggested that AtEXPA4 might exert the greatest effect from the 5 th day to the 7th day after seed germination. For AtEXPB5, the primary root growth rates of atexpb5 and $A t E X P B 5^{O E}$ were not significantly different from that of wild-type (Figure S9C). Taken together, AtEXPA4 could promote the elongation of primary root and root meristem during root growth and development, but AtEXPB5 had no effect on the growth of primary root.

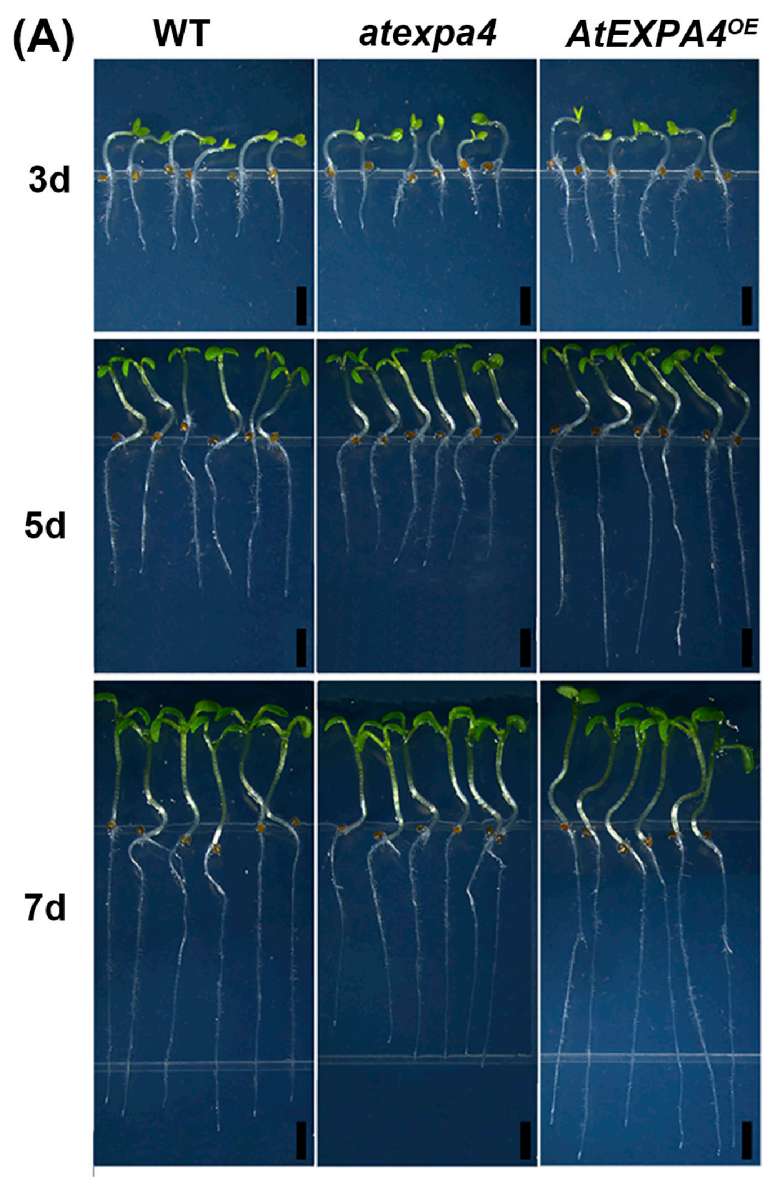

(B)
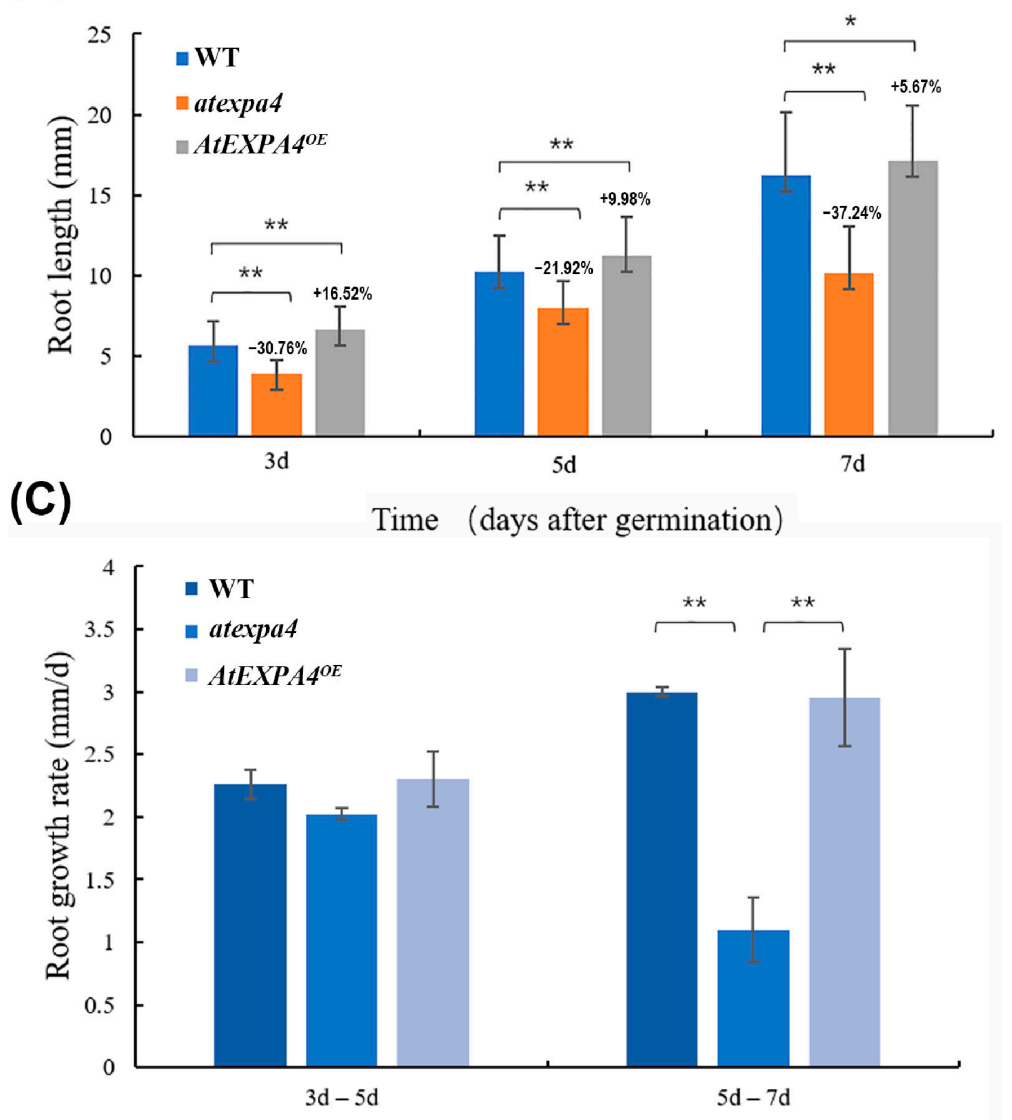

Figure 6. AtEXPA4 positively regulates primary root elongation. (A) AtEXPA4 ${ }^{O E}$ and atexpa4 seedlings germinated and grown for 3, 5, and 7 days, scale bars, $2 \mathrm{~mm}$. (B) Root lengths in (A). (C) Root growth rates from the 3rd day to the 5th day and from the 5 th day to the 7 th day after seed germination. Values are means, error bars are $\mathrm{SD}, n \geq 25$ seedlings per replicate, three biological replicates, $t$-tests as ${ }^{* *} p<0.01$ and ${ }^{*} p<0.05$. 


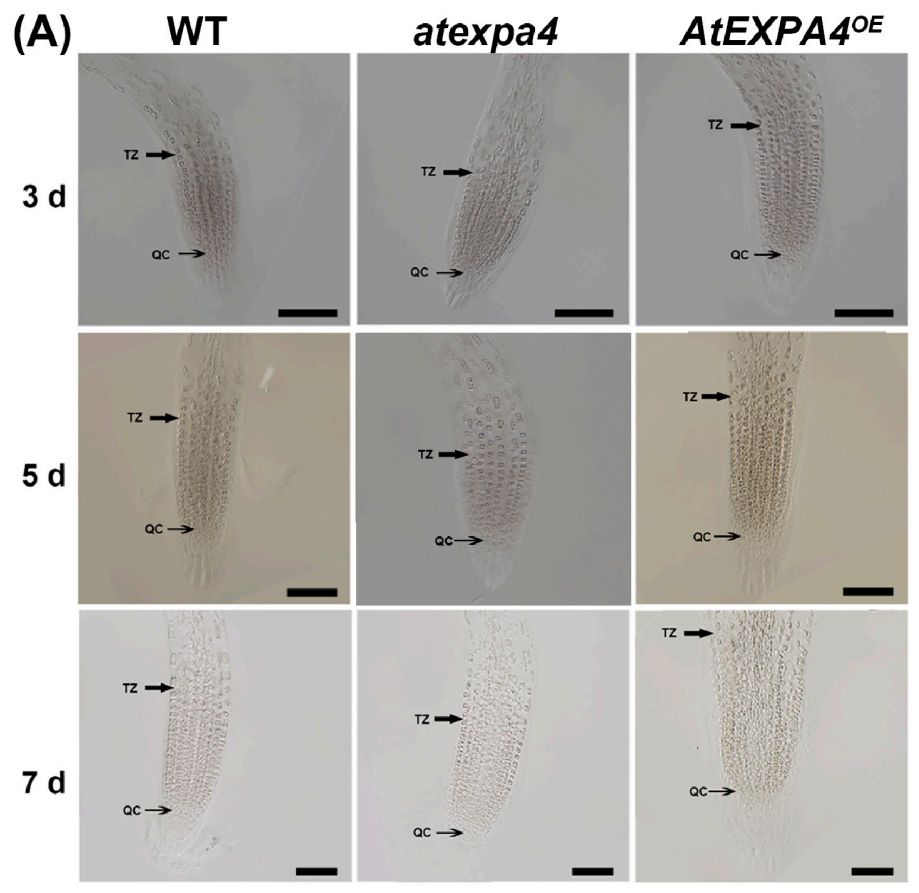

(B)

(C)
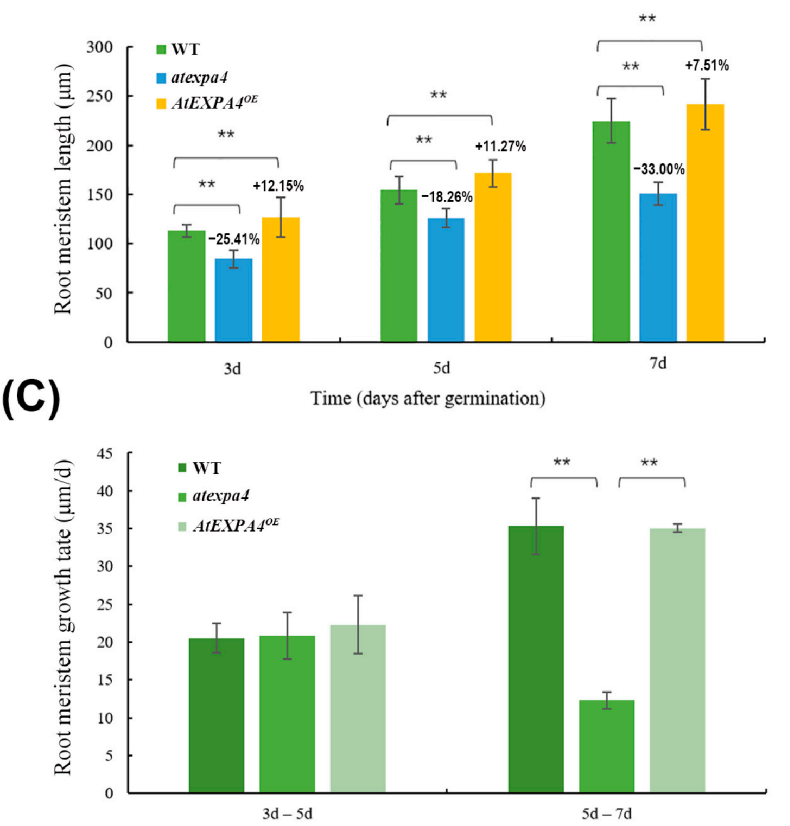

Figure 7. AtEXPA4 positively regulates root meristem length. (A) Differential interference images of AtEXPA4 ${ }^{O E}$ and atexpa4 root meristem boundary after seed germinated for 3, 5, and 7 days, scale bars, $100 \mu \mathrm{m}$. (B) Root meristem lengths in (A). (C) Root meristem growth rates from the 3 rd day to the 5 th day and from the 5 th day to the 7 th day after seed germination. Values are means, error bars are SD, $n \geq 25$ seedlings per replicate, three biological replicates, $t$-tests as ${ }^{* *} p<0.01$.

\section{Discussion}

During pollen tube growth, cell walls provide mechanical strength resisting turgor pressure to protect two sperm cells [46]. Numerous cell wall synthesis and remodeling genes have been reported in this process. In terms of cell wall synthesis, mutations of pollen-expressed $A$. thaliana cellulose synthase-like D genes CSLD1 and CSLD4 caused significant reduction in cellulose deposition of pollen tube wall, which disrupted the genetic transmission of male gametophytes [3]. Moreover, two putative $A$. thaliana galacturonosyltransferase genes GAUT13 and GAUT14 were essential for pollen tube growth [47]. Changes in cell wall assembly are relevant to pollen tube mechanical properties. For example, suppressing the expression of four leucine-rich repeat extensin genes (LRX8-11) compromised pollen germination and pollen tube growth [46,48]. In terms of cell wall remodeling, pectin methylesterase (PME) demethylates pectin to maintain stable growth of pollen tubes. Mutations of PME genes, such as VGD1 [9], AtPPME1 [49], and AtPME48 [6], showed retarded pollen tube growth in vivo and in vitro. Additionally, PME inhibitors (PMEIs) are thought to be key regulators of cell wall stability at the tip of pollen tube. Suppressing the expression of Brassica oleracea PMEI1 resulted in partial male sterility and decreased seed set by inhibition of pollen tube growth [50]. Furthermore, polygalacturonase, which leads to degradation of pectin and decomposition of cell walls, has also been confirmed to play an important role in the development of pollen tubes [7,51]. Expansins, as a type of cell wall remodeling proteins, can respond to the rapid expansion of cell walls and affect pollen tube growth [52]. Our results confirmed that the expressions of AtEXPA4 and AtEXPB5 were significantly high in mature pollen grains and pollen tubes. Moreover, proteins coding by these two genes were located on the cell wall and in the cytoplasm, and this was consistent with the positioning result of a soybean expansin protein GmEXPB2 [53]. Two single mutants, atexpa4 and atexpb5, did not exhibit any observable defects in pollens and pollen tubes, but atexpa4expb5 mutant was defective in pollen tube elongation. Moreover, the results of complementary experiments showed that whether transformed the double mutants with AtEXPA4 or AtEXPB5 could complement the slow pollen tube 
growth. However, the overexpression of AtEXPA4 and AtEXPB5 did not affect pollen tube growth. We speculate that this may be because the highest multiple of $A t E X P A 4^{O E}$ and $A t E X P B 5^{O E}$ overexpression line does not exceed 40 folds, which may not be enough to accelerate the rate of pollen tube elongation. Additionally, although the pollen germination rate of the double mutants decreased, the mutation of AtEXPA4 and AtEXPB5 did not affect the pollen competitiveness or the normal development of siliques. Taken together, AtEXPA4 and AtEXPB5 only showed redundant functions in pollen tube growth.

Interestingly, there are abundant MYB binding sites on promoters of AtEXPA4 and AtEXPB5 (Figure S10), which indicates that MYB transcription factors may participate in pollen tube and root growth by regulating expressions of AtEXPA4 and AtEXPB5. A previous study showed that a R2R3 MYB factor, TDF1, affected tapetum development by directly binding to AtEXPB5 promoter and co-regulated AtEXPB5 with another transcription factor AMS [54]. Thence, AtEXPB5 might play a role in tapetum and pollen exine development. However, there was no defect in exine of atexpb5 pollens, suggesting that TDF1 regulated the development of tapetum by acting on other cell wall remodeling genes. Pollen tube germination usually starts from the intine of mature pollen grains [6]. AtEXPA4 and AtEXPB5 were strongly expressed in mature pollen grains and pollen tubes, and simultaneous mutation of them led to obstruction of pollen tube elongation, implying that they might affect pollen tube growth by regulating the development of pollen intine. Moreover, AtMYB4, AtMYB32, AtMYB97, AtMYB101, and AtMYB120 can participate in pollen and pollen tube development $[55,56]$, suggesting that they may regulate expressions of AtEXPA4 and AtEXPB5, but further evidence is needed.

Some cell wall-related genes that affect pollen tube growth also play roles in root elongation. For example, a rhamnogalacturonan II xylosyltransferase (RG-II) gene, MGD4, participated in the growth of pollen tube and root by acting on pectic RG-II biosynthesis pathway [5]. In this study, qRT-PCR and GUS analyses also showed that AtEXPA4 was highly expressed in roots. Furthermore, it was confirmed that AtEXPA4 affected primary root elongation positively by comparing the primary root length of atexpa 4 and $A t E X P A 4^{O E}$ at different time points after seed germination. In addition, the growth rate of atexpa4 primary root sharply decreased from the 5th day to the 7th day after germination, suggesting that AtEXPA4 exerted the greatest effect during this period. We speculated that this effect might be related to the gradual increase of AtEXPA4 expression with the growth and development of primary roots. (Figure 1D). However, the mutation and overexpression of AtEXPB5 did not affect the growth and development of the primary roots (Figure S9). This indicates that AtEXPA4 plays a major role in the growth and development of primary roots, and AtEXPB5 does not affect root growth because of its extremely low expression in roots. Generally, abnormal root system also affects the response of plants to abiotic stress. The overexpression of Triticum aestivum EXPB23 (TaEXPB23) showed increased lateral roots and higher root biomass, as well as enhanced drought tolerance [57]. Additionally, the analysis of AtEXPA4 and AtEXPB5 promoters showed that they both contained abundant response elements of abscisic acid, ethylene, and jasmonic acid (Figure S10), suggesting that they might participate in plant responses to abiotic stress. Further research is needed to confirm this.

\section{Conclusions}

We isolated and characterized two expansin genes, AtEXPA4 and AtEXPB5, which are strongly expressed in mature pollens and pollen tubes. The molecular functions of AtEXPA4 and AtEXPB5 were analyzed by CRISPR/Cas9-mediated knockout and selfpromoter-mediated overexpression. The results indicated that AtEXPA4 and AtEXPB5 are redundantly required for pollen tube growth. This enriches the role of expansins in the reproductive development of plants, and also shows that the genes of EXPA and EXPB subfamily can coordinate with each other to regulate plant growth and development. Furthermore, AtEXPA4 also showed a higher expression level in roots. Based on the statistics of primary root length and root meristem size, we found that AtEXPA4 has 
a positive effect on the growth of primary roots. This also provides evidence for the involvement of expansin in the growth and development of plant roots. In addition, since there are many MYB binding sites and a variety of phytohormone response elements in the promoters of AtEXPA4 and AtEXPB5, future research can start from these aspects to uncover the regulatory pathways of expansin genes on plant growth and development.

Supplementary Materials: The following are available online at https:/ / www.mdpi.com/2073-4 425/12/2/249/s1, Figure S1: Amino acid sequences of AtEXPA4 and AtEXPB5. The blue, red, and green letters represent signal peptide, DPBB_1 domain, and Pollen_allerg_1 domain, respectively. Figure S2: Subcellular localizations of AtEXPA4::eGFP and AtEXPB5::eGFP fusion proteins in tobacco leaf epidermal cells. Figure S3: Structures of CRISPR/Cas9 (A) and AtEXPA4 (B) AtEXPB5 (C) overexpression binary vectors for Arabidopsis thaliana transformation by floral dip method. The hSpCas9 cassette is driven by $Y A O$ promoter, while sgRNAs are controlled by AtU6-26 promoters. NLS, nuclear localization sequence. Figure S4: Sequence alignment of atexpa 4 and atexpb5 mutants showed evidence of successful gene editing in the target regions, respectively. Figure S5: Sequence alignment of atexpa4expb5 mutants showed that the lines with both AtEXPA4 and AtEXPB5 knockout sites were successfully screened. (A), Detection of editing sites of AtEXPA4. (B) Detection of editing sites of AtEXPB5. Figure S6: Morphological observation of transgenic plants and floral organs. The first row is the overall shape of transgenic plants, scale bars, $4 \mathrm{~cm}$, and the upper right corners are corresponding siliques, scale bars, $4 \mathrm{~mm}$. The second row is the overall shape of flowers. The third row is the shape of pistils and stamens, scale bars, $1 \mathrm{~mm}$. Figure S7: Pollen morphology in atexpa4, atexpb5, atexpa4expb5, AtEXPA4 ${ }^{\mathrm{OE}}$, and $A t E X P B 5^{\mathrm{OE}}$. Cytological staining observation includes Alexander staining, scale bars, $100 \mu \mathrm{m}$, DAPI staining, scale bars, $50 \mu \mathrm{m}$, and aniline blue staining, scale bars, $50 \mu \mathrm{m}$. SEM is used to observe pollen grain morphology and surface decoration, scale bars, $40 \mu \mathrm{m}$. Values are means, error bars are $\mathrm{SD}, n=12$ pistils per replicate, three biological replicates. Figure S8: Overexpression of AtEXPA4 and AtEXPB5 did not affect pollen elongation. (A) Aniline blue staining of pollen tubes at $3 \mathrm{~h}, 12 \mathrm{~h}$, and $24 \mathrm{~h}$ after pollination in $A t E X P A 4^{O E}$ lines and $A t E X P B 5^{O E}$ lines, hours (h). Asterisks show positions where pollen tubes arrive, scale bars, $300 \mu \mathrm{m}$. (B) the percentage of pollen tube to pistil length in (A). Values are means, error bars are $\mathrm{SD}, n=12$ pistils per replicate, three biological replicates. Figure S9: AtEXPB5 does not affect the primary root elongation. (A) AtEXPB5 OE and atexpb5 seedlings germinated and grown for 3, 5, and 7 days, scale bars, $2 \mathrm{~mm}$. (B) Root lengths in (A). (C) Root growth rates from the 3rd day to the 5th day and from the 5th day to the 7th day after seed germination. Values are means, error bars are $\mathrm{SD}, \mathrm{n} \geq 12$ seedlings per replicate, three biological replicates. Figure S10: Distribution of cis-acting elements in the $1.5 \mathrm{~kb}$ upstream promoter regions of AtEXPA4 and AtEXPB5. The different types of cis-acting elements are represented by different colors. The scale above was to measure nucleotides length. The results are predicted by the PlantCARE website (http://bioinformatics.psb.ugent.be/webtools/plantcare/html/). Table S1. Primers used in this study. Table S2. Off-target detection of atexpa4. Table S3. Off-target detection of atexpb5.

Author Contributions: Conceptualization, W.L. and J.C.; methodology, W.L.; validation, W.L. and L.X.; formal analysis, W.L. and L.X.; investigation, W.L. and H.L.; resources, J.C.; data curation, W.L.; writing—original draft preparation, W.L.; writing—review and editing, L.X. and J.C.; visualization, W.L. and H.L.; supervision, J.C.; project administration, W.L. and J.C.; funding acquisition, J.C. All authors have read and agreed to the published version of the manuscript.

Funding: This research was funded by grant from the National Natural Science Foundation of China (No. 31772311).

Institutional Review Board Statement: Not applicable.

Informed Consent Statement: Not applicable.

Data Availability Statement: Data sharing not applicable.

Acknowledgments: Thanks to Nianhang Rong from the Bio-ultrastructure Analysis Laboratory of Analysis center of Agrobiology and Environmental Sciences in Zhejiang University for supporting the use of scanning electron microscopy in this study.

Conflicts of Interest: The authors declare no conflict of interest. 


\section{References}

1. Vaahtera, L.; Schulz, J.; Hamann, T. Cell wall integrity maintenance during plant development and interaction with the environment. Nat. Plants 2019, 5, 924-932. [CrossRef] [PubMed]

2. Kuo, K.H.; Shih, J.J.; Liao, Y.H.; Fu, T.W.; Fan, L.J.; Yang, Y.W.; Lin, J.L. Thermal decomposition of HSCH2CH2OH on Cu(111): Identification and adsorption geometry of surface intermediates. J. Phys. Chem. B 2005, 109, 5055-5059. [CrossRef] [PubMed]

3. Wang, W.; Wang, L.; Chen, C.; Xiong, G.; Tan, X.Y.; Yang, K.Z.; Wang, Z.C.; Zhou, Y.; Ye, D.; Chen, L.Q. Arabidopsis CSLD1 and CSLD4 are required for cellulose deposition and normal growth of pollen tubes. J. Exp. Bot. 2011, 62, 5161-5177. [CrossRef] [PubMed]

4. Hu, H.; Zhang, R.; Dong, S.; Li, Y.; Fan, C.; Wang, Y.; Xia, T.; Chen, P.; Wang, L.; Feng, S.; et al. AtCSLD3 and GhCSLD3 mediate root growth and cell elongation downstream of the ethylene response pathway in Arabidopsis. J. Exp. Bot. 2018, 69, 1065-1080. [CrossRef] [PubMed]

5. $\quad$ Liu, X.L.; Liu, L.; Niu, Q.K.; Xia, C.; Yang, K.Z.; Li, R.; Chen, L.Q.; Zhang, X.Q.; Zhou, Y.; Ye, D. Male gametophyte defective 4 encodes a rhamnogalacturonan II xylosyltransferase and is important for growth of pollen tubes and roots in Arabidopsis. Plant $J$. 2011, 65, 647-660. [CrossRef]

6. Leroux, C.; Bouton, S.; Kiefer-Meyer, M.C.; Fabrice, T.N.; Mareck, A.; Guénin, S.; Fournet, F.; Ringli, C.; Pelloux, J.; Driouich, A.; et al. PECTIN METHYLESTERASE48 is involved in Arabidopsis pollen grain germination. Plant Physiol. 2015, 167, 367-380. [CrossRef]

7. Huang, L.; Cao, J.; Zhang, A.; Ye, Y.; Zhang, Y.; Liu, T. The polygalacturonase gene BcMF2 from Brassica campestris is associated with intine development. J. Exp. Bot. 2009, 60, 301-313. [CrossRef]

8. Hu, H.; Zhang, R.; Feng, S.; Wang, Y.; Wang, Y.; Fan, C.; Li, Y.; Liu, Z.; Schneider, R.; Xia, T.; et al. Three AtCesA6-like members enhance biomass production by distinctively promoting cell growth in Arabidopsis. Plant Biotechnol. J. 2018, 16, 976-988. [CrossRef]

9. Jiang, L.; Yang, S.L.; Xie, L.F.; Puah, C.S.; Zhang, X.Q.; Yang, W.C.; Sundaresan, V.; Ye, D. VANGUARD1 encodes a pectin methylesterase that enhances pollen tube growth in the Arabidopsis style and transmitting tract. Plant Cell 2005, 17, 584-596. [CrossRef]

10. McQueen-Mason, S.; Cosgrove, D.J. Disruption of hydrogen bonding between plant cell wall polymers by proteins that induce wall extension. Proc. Natl. Acad. Sci. USA 1994, 91, 6574. [CrossRef]

11. Cosgrove, D.J. Growth of the plant cell wall. Nat. Rev. Mol. Cell Biol. 2005, 6, 850-861. [CrossRef]

12. Liu, W.; Lyu, T.; Xu, L.; Hu, Z.; Xiong, X.; Liu, T.; Cao, J. Complex Molecular Evolution and Expression of Expansin Gene Families in Three Basic Diploid Species of Brassica. Int. J. Mol. Sci. 2020, 21, 3424. [CrossRef] [PubMed]

13. Castillo, R.M.; Mizuguchi, K.; Dhanaraj, V.; Albert, A.; Blundell, T.L.; Murzin, A.G. A six-stranded double-psi beta barrel is shared by several protein superfamilies. Structure 1999, 7, 227-236. [CrossRef]

14. Mizuguchi, K.; Dhanaraj, V.; Blundell, T.L.; Murzin, A.G. N-ethylmaleimide-sensitive fusion protein (NSF) and CDC48 confirmed as members of the double-psi beta-barrel aspartate decarboxylase/formate dehydrogenase family. Structure 1999, 7, R215-R216. [CrossRef]

15. Cosgrove, D.J. Plant expansins: Diversity and interactions with plant cell walls. Curr. Opin. Plant Biol. 2015, $25,162-172$. [CrossRef] [PubMed]

16. Kende, H.; Bradford, K.; Brummell, D.; Cho, H.T.; Cosgrove, D.; Fleming, A.; Gehring, C.; Lee, Y.; McQueen-Mason, S.; Rose, J.; et al. Nomenclature for members of the expansin superfamily of genes and proteins. Plant Mol. Biol. 2004, 55, 311-314. [CrossRef] [PubMed]

17. Chen, Y.; Zhang, B.; Li, C.; Lei, C.; Kong, C.; Yang, Y.; Gong, M. A comprehensive expression analysis of the expansin gene family in potato (Solanum tuberosum) discloses stress-responsive expansin-like B genes for drought and heat tolerances. PLoS ONE 2019, 14, e0219837. [CrossRef] [PubMed]

18. Han, Z.; Liu, Y.; Deng, X.; Liu, D.; Liu, Y.; Hu, Y.; Yan, Y. Genome-wide identification and expression analysis of expansin gene family in common wheat (Triticum aestivum L.). BMC Genom. 2019, 20, 101. [CrossRef] [PubMed]

19. Guimaraes, L.A.; Mota, A.P.Z.; Araujo, A.C.G.; de Alencar Figueiredo, L.F.D.; Pereira, B.M.; de Passos Saraiva, M.A.; Silva, R.B.; Danchin, E.G.J.; Guimaraes, P.M.; Brasileiro, A.C.M. Genome-wide analysis of expansin superfamily in wild Arachis discloses a stress-responsive expansin-like B gene. Plant Mol. Biol. 2017, 94, 79-96. [CrossRef]

20. Yan, A.; Wu, M.; Yan, L.; Hu, R.; Ali, I.; Gan, Y. AtEXP2 is involved in seed germination and abiotic stress response in Arabidopsis. PLoS ONE 2014, 9, e85208. [CrossRef]

21. Xu, H.; Lantzouni, O.; Bruggink, T.; Benjamins, R.; Lanfermeijer, F.; Denby, K.; Schwechheimer, C.; Bassel, G.W. A Molecular Signal Integration Network Underpinning Arabidopsis Seed Germination. Curr. Biol. CB 2020, 30, 3703-3712.e3704. [CrossRef] [PubMed]

22. Sánchez-Montesino, R.; Bouza-Morcillo, L.; Marquez, J.; Ghita, M.; Duran-Nebreda, S.; Gómez, L.; Holdsworth, M.J.; Bassel, G.; Oñate-Sánchez, L. A Regulatory Module Controlling GA-Mediated Endosperm Cell Expansion Is Critical for Seed Germination in Arabidopsis. Mol. Plant 2019, 12, 71-85. [CrossRef]

23. Park, C.H.; Kim, T.W.; Son, S.H.; Hwang, J.Y.; Lee, S.C.; Chang, S.C.; Kim, S.H.; Kim, S.W.; Kim, S.K. Brassinosteroids control AtEXPA5 gene expression in Arabidopsis thaliana. Phytochemistry 2010, 71, 380-387. [CrossRef] 
24. Son, S.H.; Chang, S.C.; Park, C.H.; Kim, S.K. Ethylene negatively regulates EXPA5 expression in Arabidopsis thaliana. Physiol. Plant. 2012, 144, 254-262. [CrossRef]

25. Ma, N.; Wang, Y.; Qiu, S.; Kang, Z.; Che, S.; Wang, G.; Huang, J. Overexpression of OsEXPA8, a root-specific gene, improves rice growth and root system architecture by facilitating cell extension. PLoS ONE 2013, 8, e75997. [CrossRef] [PubMed]

26. Wang, Y.; Ma, N.; Qiu, S.; Zou, H.; Zang, G.; Kang, Z.; Wang, G.; Huang, J. Regulation of the $\alpha$-expansin gene OsEXPA8 expression affects root system architecture in transgenic rice plants. Mol. Breed. 2014, 34, 47-57. [CrossRef]

27. Lee, H.W.; Kim, J. EXPANSINA17 up-regulated by LBD18/ASL20 promotes lateral root formation during the auxin response Plant Cell Physiol. 2013, 54, 1600-1611. [CrossRef]

28. Lee, H.W.; Kim, M.J.; Kim, N.Y.; Lee, S.H.; Kim, J. LBD18 acts as a transcriptional activator that directly binds to the EXPANSIN14 promoter in promoting lateral root emergence of Arabidopsis. Plant J. 2013, 73, 212-224. [CrossRef] [PubMed]

29. Valdivia, E.R.; Sampedro, J.; Lamb, J.C.; Chopra, S.; Cosgrove, D.J. Recent proliferation and translocation of pollen group 1 allergen genes in the maize genome. Plant Physiol. 2007, 143, 1269-1281. [CrossRef]

30. Cosgrove, D.J. Relaxation in a high-stress environment: The molecular bases of extensible cell walls and cell enlargement. Plant Cell 1997, 9, 1031-1041. [CrossRef]

31. Valdivia, E.R.; Wu, Y.; Li, L.C.; Cosgrove, D.J.; Stephenson, A.G. A group-1 grass pollen allergen influences the outcome of pollen competition in maize. PLoS ONE 2007, 2, e154. [CrossRef] [PubMed]

32. Mollet, J.C.; Leroux, C.; Dardelle, F.; Lehner, A. Cell Wall Composition, Biosynthesis and Remodeling during Pollen Tube Growth. Plants (Basel) 2013, 2, 107-147. [CrossRef]

33. Qin, Y.; Leydon, A.R.; Manziello, A.; Pandey, R.; Mount, D.; Denic, S.; Vasic, B.; Johnson, M.A.; Palanivelu, R. Correction: Penetration of the Stigma and Style Elicits a Novel Transcriptome in Pollen Tubes, Pointing to Genes Critical for Growth in a Pistil. PLoS Genet. 2016, 12, e1006210. [CrossRef]

34. Winter, D.; Vinegar, B.; Nahal, H.; Ammar, R.; Wilson, G.V.; Provart, N.J. An “Electronic Fluorescent Pictograph" browser for exploring and analyzing large-scale biological data sets. PLoS ONE 2007, 2, e718. [CrossRef] [PubMed]

35. Swanson, R.; Clark, T.; Preuss, D. Expression profiling of Arabidopsis stigma tissue identifies stigma-specific genes. Sex. Plant Reprod. 2005, 18, 163-171. [CrossRef]

36. Qin, Y.; Leydon, A.R.; Manziello, A.; Pandey, R.; Mount, D.; Denic, S.; Vasic, B.; Johnson, M.A.; Palanivelu, R. Penetration of the stigma and style elicits a novel transcriptome in pollen tubes, pointing to genes critical for growth in a pistil. PLoS Genet. 2009, 5, e1000621. [CrossRef]

37. Clough, S.J.; Bent, A.F. Floral dip: A simplified method for Agrobacterium-mediated transformation of Arabidopsis thaliana. Plant J. 1998, 16, 735-743. [CrossRef] [PubMed]

38. Xiong, X.; Liu, W.; Jiang, J.; Xu, L.; Huang, L.; Cao, J. Efficient genome editing of Brassica campestris based on the CRISPR/Cas9 system. Mol. Genet. Genom. 2019, 294, 1251-1261. [CrossRef]

39. Bowman, J.L.; Drews, G.N.; Meyerowitz, E.M. Expression of the Arabidopsis floral homeotic gene AGAMOUS is restricted to specific cell types late in flower development. Plant Cell 1991, 3, 749-758. [CrossRef]

40. Yamaoka, Y.; Yu, Y.; Mizoi, J.; Fujiki, Y.; Saito, K.; Nishijima, M.; Lee, Y.; Nishida, I. PHOSPHATIDYLSERINE SYNTHASE1 is required for microspore development in Arabidopsis thaliana. Plant J. 2011, 67, 648-661. [CrossRef] [PubMed]

41. Alexander, M.P. Differential staining of aborted and nonaborted pollen. Stain Technol. 1969, 44, 117-122. [CrossRef]

42. McCormick, S. Control of male gametophyte development. Plant Cell 2004, 16, S142-S153. [CrossRef]

43. Zhang, Z.B.; Zhu, J.; Gao, J.F.; Wang, C.; Li, H.; Li, H.; Zhang, H.Q.; Zhang, S.; Wang, D.M.; Wang, Q.X.; et al. Transcription factor AtMYB103 is required for anther development by regulating tapetum development, callose dissolution and exine formation in Arabidopsis. Plant J. 2007, 52, 528-538. [CrossRef] [PubMed]

44. Lin, S.; Dong, H.; Zhang, F.; Qiu, L.; Wang, F.; Cao, J.; Huang, L. BcMF8, a putative arabinogalactan protein-encoding gene, contributes to pollen wall development, aperture formation and pollen tube growth in Brassica campestris. Ann. Bot. 2014, 113, 777-788. [CrossRef]

45. Yan, L.; Wei, S.; Wu, Y.; Hu, R.; Li, H.; Yang, W.; Xie, Q. High-Efficiency Genome Editing in Arabidopsis Using YAO PromoterDriven CRISPR/Cas9 System. Mol. Plant 2015, 8, 1820-1823. [CrossRef]

46. Wang, X.; Wang, K.; Yin, G.; Liu, X.; Liu, M.; Cao, N.; Duan, Y.; Gao, H.; Wang, W.; Ge, W.; et al. Pollen-Expressed Leucine-Rich Repeat Extensins Are Essential for Pollen Germination and Growth. Plant Physiol. 2018, 176, 1993-2006. [CrossRef]

47. Wang, L.; Wang, W.; Wang, Y.Q.; Liu, Y.Y.; Wang, J.X.; Zhang, X.Q.; Ye, D.; Chen, L.Q. Arabidopsis galacturonosyltransferase (GAUT) 13 and GAUT14 have redundant functions in pollen tube growth. Mol. Plant 2013, 6, 1131-1148. [CrossRef]

48. Sede, A.R.; Borassi, C.; Wengier, D.L.; Mecchia, M.A.; Estevez, J.M.; Muschietti, J.P. Arabidopsis pollen extensins LRX are required for cell wall integrity during pollen tube growth. FEBS Lett. 2018, 592, 233-243. [CrossRef]

49. Tian, G.W.; Chen, M.H.; Zaltsman, A.; Citovsky, V. Pollen-specific pectin methylesterase involved in pollen tube growth. Dev. Biol. 2006, 294, 83-91. [CrossRef]

50. Zhang, G.Y.; Feng, J.; Wu, J.; Wang, X.W. BoPMEI1, a pollen-specific pectin methylesterase inhibitor, has an essential role in pollen tube growth. Planta 2010, 231, 1323-1334. [CrossRef] [PubMed]

51. Huang, L.; Ye, Y.; Zhang, Y.; Zhang, A.; Liu, T.; Cao, J. BcMF9, a novel polygalacturonase gene, is required for both Brassica campestris intine and exine formation. Ann. Bot. 2009, 104, 1339-1351. [CrossRef] [PubMed] 
52. Valdivia, E.R.; Stephenson, A.G.; Durachko, D.M.; Cosgrove, D. Class B beta-expansins are needed for pollen separation and stigma penetration. Sex. Plant Reprod. 2009, 22, 141-152. [CrossRef] [PubMed]

53. Guo, W.; Zhao, J.; Li, X.; Qin, L.; Yan, X.; Liao, H. A soybean $\beta$-expansin gene GmEXPB2 intrinsically involved in root system architecture responses to abiotic stresses. Plant J. 2011, 66, 541-552. [CrossRef] [PubMed]

54. Lou, Y.; Zhou, H.S.; Han, Y.; Zeng, Q.Y.; Zhu, J.; Yang, Z.N. Positive regulation of AMS by TDF1 and the formation of a TDF1-AMS complex are required for anther development in Arabidopsis thaliana. New Phytol. 2018, 217, 378-391. [CrossRef] [PubMed]

55. Preston, J.; Wheeler, J.; Heazlewood, J.; Li, S.F.; Parish, R.W. AtMYB32 is required for normal pollen development in Arabidopsis thaliana. Plant J. 2004, 40, 979-995. [CrossRef] [PubMed]

56. Liang, Y.; Tan, Z.M.; Zhu, L.; Niu, Q.K.; Zhou, J.J.; Li, M.; Chen, L.Q.; Zhang, X.Q.; Ye, D. MYB97, MYB101 and MYB120 function as male factors that control pollen tube-synergid interaction in Arabidopsis thaliana fertilization. PLoS Genet. 2013, 9, e1003933. [CrossRef]

57. Li, A.X.; Han, Y.Y.; Wang, X.; Chen, Y.H.; Zhao, M.R.; Zhou, S.-M.; Wang, W. Root-specific expression of wheat expansin gene TaEXPB23 enhances root growth and water stress tolerance in tobacco. Environ. Exp. Bot. 2015, 110, 73-84. [CrossRef] 